Ann. Biol. anim. Bioch. Biophys., 1977, 17 (4), 559-565.

\title{
Détermination de la valeur alimentaire de l'urée donnée en perfusion intracæcale chez le lapin
}

\author{
par A. SALSE, F. CRAMPES, P. RAYNAUD.
}

Instifut de Physiologie, 2 rue François-Magendie, 31400 Toulouse.

Summary. Measurement of $\mathrm{N}$ urea dietary value by intracaecal perfusion in rabbit.

The dietary value of urea as a nitrogenous supply perfused into the cecum was studied in rabbits. A diet containing 8 p. 100 prolein was more efficient when supplemented with urea perfusion. The utilization coefficient $(9 \pm 5)$ was thus lower than with a diet containing 16 p. 100 protein $(40 \pm 8)$; there was no significant difference between the proiein efficiency coefficients $(7 \pm 4$ and $9 \pm 2$ ).

Examination of nitrogen urinary secretion in the different experiments suggests that at least half the ureic nitrogen perfused was retained by the rabbit and thus seemed more efficient than the 16 p. 100 protein diet.

The quantity of bacteria in the soft feces, relatively low with the 8 p. 100 protein diet, was as great when the latter diet was supplemented with intracaecal perfused urea as when animals were fed a 16 p. 100 protein diet.

Les recherches qui ont été effectuées pour mieux connaître le métabolisme des substances azotées dans le cæcum du lapin (Marty, 1971), font ressortir que les processus de protéolyse et de protéosynthèse des bactéries passent le plus souvent par le stade azote ammoniacal. D'ailleurs, celui-ci peut être utilisé par les bactéries du cæcum comme seule source azotée, qu'il provienne indifféremment de substances protéiques ou de produits comme l'urée.

On peuł donc considérer, indépendamment d'autres facteurs, que l'intensité de la prolifération microbienne cæcale dans le tractus digestif, dépend du taux d'azote ammoniacal disponible avec toutefois une limite au-delà de laquelle il franchit la barrière digestive pour se retrouver dans le sang et déterminer des symptômes d'intoxication.

De plus, les protéines bactériennes dont la composition en acides aminés est remarquablement constante et indépendante du régime alimentaire (Fuller et Moore, 1971), constituent une source protéique de bonne qualité (Proto et Gianani, 1969 ; Yoshida ef al., 1971), susceptible de couvrir les besoins azotés du lapin. Ainsi, par l'intermédiaire de la protéosynthèse microbienne importante qui s'effectue dans le 
cæcum à partir de l'azote ammoniacal et grâce à la récupération des protéines bactériennes par le phénomène de la cæcotrophie (Barnes, Fiala et Kwong, 1963 ; Proto, 1965 ; Battaglini, 1968), et celui de la lyse colique (Bonnafous, 1973), le lapin pourra, en définitive utiliser des sources azotées non protéiques telle que l'urée (Slade et Robinson, 1970 ; Hoover et Heitmann 1975 ; Niedzwiadek, 1976).

Ceci nous a amené à étudier la valeur nutritionnelle d'une telle source d'azote pour le lapin.

Afin d'éviter la réabsorption et les processus uréasiques qui se manifestent dans les parties antérieures du tube digestif, nous pratiquons des perfusions continues de solutions d'urée directement dans le cæcum. De cette façon, l'ammoniac libéré par les uréases bactériennes, peut être utilisé sur place.

Cependant, si l'azote ammoniacal est produif en excès par rapport à la capacité de protéosynthèse de la population bactérienne, il s'accumule dans le cæcum et passe dans le sang ; il est capté par le foie et retransformé en urée qui est excrétée dans les urines, exception faiłe de la fraction qui peut être recyclée après un nouveau passage dans le tube digestif. Cela peut être considéré évidemment comme une perte de constituants azotés, mais aussi comme un gaspillage d'énergie utilisée pour la synthèse et l'excrétion de l'urée (Chalupa et al., 1970).

Enfin, lorsque la concentration en azote ammoniacal dans le cæcum devient excessive, le foie est « débordé »; l'ammoniac passe dans le sang périphérique et délermine des accidents de toxicité dont la gravité croît avec la concentration et qui peuvent entraîner la mort rapide de l'animal (Hsia, 1974). En fait, on ne connaît pas les taux d'azote ammoniacal que le lapin peut tolérer. Il nous a donc paru nécessaire de déterminer avant tout, les potentialités toxiques de l'urée et d'établir les doses maximales et optimales perfusables, suivani nos conditions expérimentales, dans le cæcum du lapin. La valeur nutritionnelle de l'urée ainsi fournie étant étudiée ensuite.

Dans une première série d'expériences, 3 lots de 3 lapins adultes nourris avec de l'aliment contenant 8 p. 100 de protéines, sont soumis à des perfusions intracæcales fournissant respectivement $1 \mathrm{~g}, 2 \mathrm{~g}$ ou $4 \mathrm{~g}$ d'urée par jour. Les $50 \mathrm{ml}$ de solution ainsi perfusés apportent, en outre, quotidiennement, $20 \mathrm{~g}$ de maltose, $0,5 \mathrm{~g}$ de mélange minéral d'Osborne et $10 \mathrm{mg}$ de soufre, cela pendant une durée de 10 jours. La consommation alimentaire, la variation de poids corporel et le bilan azoté sont calculés pour chaque animal et exprimés en g par $24 \mathrm{~h}$.

\section{TABLEAU 1}

Influence de la dose d'urée perfusée sur la consommation alimentaire, le poids corporel $(\Delta \mathrm{P})$ ef le bilan azoté $(\Delta N)$

\begin{tabular}{|c|c|c|c|}
\hline & $\begin{array}{l}\text { Consommation } \\
\text { en } g / 24 h\end{array}$ & $\begin{array}{c}\Delta P \\
\text { en } g / 24 h\end{array}$ & $\begin{array}{l}\Delta N \\
\text { en } g / 24 h\end{array}$ \\
\hline $\begin{array}{l}1 \mathrm{~g} \text { urée } \ldots \ldots \ldots \ldots \\
2 \mathrm{~g} \text { urée } \ldots \ldots \ldots \ldots \\
4 \mathrm{~g} \text { urée } \ldots \ldots \ldots \ldots\end{array}$ & $\begin{array}{r}102 \pm 14 \\
150 \pm 20 \\
90 \pm 18\end{array}$ & $\begin{array}{l}+15 \pm 8 \\
+20 \pm 14 \\
+\quad 4 \pm 7\end{array}$ & $\begin{array}{l}+0,20 \pm 0,07 \\
+0,21 \pm 0,17 \\
+0,06 \pm 0,07\end{array}$ \\
\hline
\end{tabular}


Les variations importantes de consommation rendent très difficile l'interprétation des résultats. Il semble cependant que la perfusion fournissant $2 \mathrm{~g}$ d'urée par jour soit la plus efficace bien que la croissance supérieure relevée pour ce lot d'animaux puisse être due uniquement à sa consommation alimentaire plus élevée.

Nous retiendrons cependant cette valeur qui permet de respecter les règles énoncées par Journet (1973) sur la supplémentation des régimes par l'azole uréique chez les ruminants (l'azote uréique peut difficilement dépasser 50 p. 100 de la ration sans toutefois excéder $0,45 \mathrm{~g} / \mathrm{kg}$ de poids vif). En effet, bien que la composition des flores digestives soił différente pour ces deux espèces (Smith, 1965 ; Smith et Crabb 1961), les phénomènes doivent rester semblables dans le cæcum du lapin et dans la panse des ruminants (Hall, 1952).

Le fait certain est que la limite tolérable, dans nos conditions expérimentales, correspond à la perfusion contenant $4 \mathrm{~g}$ d'urée par jour. Nous avons montré que 2 lapins soumis immédiatement à cette dose sont morts dans les 48 h suivant le début de l'expérience et nous n'avons pu faire supporter les $4 \mathrm{~g}$ d'urée/jour à des lapins adultes qu'à la condition de les « adapter » préalablement avec des perfusions moins concentrées pendant 10 à 15 jours.

Une deuxième série d'expériences est conduite sur un ensemble de 28 lapins adultes, de race commune, répartis en 4 lots :

- les animaux du premier groupe sont nourris ad libitum avec de l'aliment contenant 8 p. 100 de protéines ;

TABLEAU 2

Influence de différents régimes alimentaires sur quelques paramètres nutritionnels $(\mathrm{n}=7)$

\begin{tabular}{|c|c|c|c|c|c|}
\hline & $\begin{array}{c}\text { A } \\
\text { Aliment } \\
8 \text { p. } 100 \\
\text { de protéines }\end{array}$ & $\begin{array}{c}\text { B } \\
\text { Aliment } \\
8 \mathrm{p} .100 \\
\text { de protéines } \\
+ \text { perfusion } \\
\text { maltose }\end{array}$ & $\begin{array}{c}\text { C } \\
\text { Aliment } \\
8 \text { p. } 100 \\
\text { de protéines } \\
+ \text { perfusion } \\
\text { maltose et urée }\end{array}$ & $\begin{array}{c}\text { D } \\
\text { Aliment } \\
16 \text { p. } 100 \\
\text { de protéines }\end{array}$ & Signification \\
\hline Azote ingéré $(g / 24 h)$ & $2,00 \pm 0,10$ & $1,75 \pm 0,36$ & $\begin{array}{c}1,63 \pm 0,31 \\
+0,93 \\
\text { (N uréique) }\end{array}$ & $3,56 \pm 0,18$ & N.S. entre $A, B$ et $C$ \\
\hline Azote urinaire $(g / 24 h) .$. & $1,20 \pm 0,09$ & $1,09 \pm 0,09$ & $1,56 \pm 0,23$ & $1,18 \pm 0,22$ & N.S. \\
\hline Azote fécal $(g / 24 h) \ldots$. & $0,90 \pm 0,08$ & $0,81 \pm 0,17$ & $0,82 \pm 0,18$ & $0,94 \pm 0,14$ & N.S. \\
\hline Bilan azoté $(g / 24 h)$ & $-0,11 \pm 0,09$ & $-0,16 \pm 0,09$ & $+0,19 \pm 0,08$ & $+1,45 \pm 0,23$ & $\begin{array}{c}\alpha<5 \text { p. } 100 \\
\text { entre C et } A \\
\alpha<1 \text { p. } 1000 \\
\text { entre } C \text { et } B\end{array}$ \\
\hline Variation poids $(g / 24 h)$ & $-0,9 \pm 2,5$ & $-4,0 \pm 3,3$ & $+15,5 \pm 6,7$ & $30,8 \pm 5,2$ & $\begin{array}{l}\alpha<5 \text { p. } 100 \\
\text { entre } C \text { et } A\end{array}$ \\
\hline $\begin{array}{l}\text { Densité bactérienne dans } \\
\text { les fèces dures }\left(10^{10} / \mathrm{g} M S\right)\end{array}$ & $8 \pm 2$ & $9 \pm 1$ & $10 \pm 3$ & $13 \pm 4$ & N.S. \\
\hline $\begin{array}{l}\text { Densité bactérienne dans } \\
\text { les fèces molles } 10\left({ }^{10} / \mathrm{g} \mathrm{MS}\right)\end{array}$ & $29 \pm 4$ & $30 \pm 2$ & $58 \pm 8$ & $62 \pm 6$ & $\begin{array}{l}\alpha<2 \text { p. } 100 \\
\text { entre } C \text { et } A\end{array}$ \\
\hline
\end{tabular}


- ceux du 2 e lot reçoivent, en outre, chaque jour en perfusion intracæcale, $50 \mathrm{ml}$ de solution contenant $10 \mathrm{~g}$ de maltose, $0,3 \mathrm{~g}$ de mélange minéral et $10 \mathrm{mg}$ de soufre à la vitesse de $6 \mathrm{ml}$ l'heure ;

- dans le 3 e lot, ils bénéficient en plus de $2 \mathrm{~g}$ d'urée dans la solution précédente ;

- le 4 e lot est nourri ad libitum avec de l'aliment contenant 16 p. 100 de protéines.

Nous contrôlons chaque jour sur ces animaux : la consommation alimentaire, le poids corporel, l'excrétion azotée urinaire et fécale. Nous calculons les bilans azotés, et des numérations bactériennes sont effectuées à la fin de l'expérimentation sur les deux types de fèces.

L'examen des résultats obtenus montre qu'il n'y a pas de différence significative pour l'azote ingéré et l'azote excrété entre les 3 premiers groupes.

Le fait que l'excrétion azotée fécale n'augmente pas lors de la perfusion intracæcale d'urée, laisse supposer que celle-ci est totalement dégradée par la flore du cæcum. L'azote ammoniacal ainsi libéré est entièrement capté soit par les bactéries pour effectuer leur protéosynthèse (comme le montre l'augmentation de la densité bactérienne dans les fèces molles des animaux recevant l'urée), soit par le sang pour reformer de l'urée ou des acides aminés non essentiels au niveau du foie (Hoover et Heiłmann, 1975).

Par contre, l'azote excrété dans les urines tend à diminuer chez les animaux recevant la perfusion de maltose seul et à augmenter chez ceux qui reçoivent l'urée.

Le maltose semble donc permettre une utilisation plus grande de l'urée endogène qui, recyclée par filtration à travers la paroi du tube digestif (Koj ef al., 1964 ; Regoeczi et al., 1965 ; Fonolla, Aguilera et Sanz, 1972) peut être mieux utilisée par les bactéries qui trouvent, dans ces conditions, l'énergie nécessaire directement disponible pour leur protéosynthèse.

D'autre part, l'augmentation de l'excrétion azotée urinaire chez les animaux recevant l'urée permet, par comparaison avec ceux qui reçoivent le maltose seul ou avec les témoins non perfusés, de penser que plus de la moitié de l'urée perfusée est effectivement retenue ; le reste est éliminé dans l'urine entraînant une augmentation de l'excrétion azotée urinaire de $0,35 \mathrm{~g}$ ou $0,47 \mathrm{~g}$ par jour, suivant le groupe avec lequel est effectuée la comparaison, alors qu'il a été fourni environ $1 \mathrm{~g}$ d'azote uréique par jour.

Mais si presque la moitié de l'urée fournie est éliminée, on peut constater que la fraction utilisée est très efficace puisque le gain de poids des lapins recevant l'urée est supérieur à celui des autres lots nourris avec 8 p. 100 de protéines et se rapproche de celui des animaux qui en consomment 16 p. 100 (Salse ef al., 1976).

Aussi, nous avons essayé de déterminer la valeur protéique de l'urée pour le lapin en comparant les Coefficients d'Utilisation Protéique, les Coefficients d'Efficacité Protéique et les Coefficients d'Efficacité Protéique modifiés calculés en faisant intervenir l'azote retenu, pour les 3 séries suivantes : - animaux soumis à un régime contenant 8 p. 100 de protéines, - animaux recevant le même régime alimentaire supplémenté par la perfusion intracæcale d'urée et - animaux nourris avec 16 p. 100 de protéines. 


\section{TABLEAU 3}

Influence de la perfusion intracæcale de $2 \mathrm{~g}$ d'urée sur les coefficients nutritionnels et comparaison avec un régime contenant 16 p. 100 de protéines

\begin{tabular}{|c|c|c|}
\hline $\begin{array}{c}\text { Aliment } \\
8 \text { p. } 100 \text { (1) }\end{array}$ & $\begin{array}{c}\text { Aliment } \\
8 \mathrm{p} \cdot 100 \\
+2 \mathrm{~g} \text { urée (2) }\end{array}$ & $\begin{array}{c}\text { Aliment } \\
16 \text { p. } 100(3)\end{array}$ \\
\hline
\end{tabular}

\begin{tabular}{lcccc} 
CUP $\ldots \ldots \ldots \ldots \ldots \ldots$ & $<0$ & $9 \pm 5$ & $40 \pm 8$ & $\begin{array}{l}\alpha<5 \text { p. } 100 \text { entre (1) et (2) } \\
\alpha<1 \text { p. 100 entre (2) et (3) }\end{array}$ \\
\hline CEP $\ldots \ldots \ldots \ldots \ldots \ldots$ & $<0$ & $7 \pm 4$ & $9 \pm 2$ & N.S. \\
\hline CEP modifié $\ldots \ldots \ldots \ldots$ & faible & $68 \pm 22$ & $24 \pm 2$ & N.S. \\
\hline
\end{tabular}

1) Les coefficients d'utilisation protéique (CUP) calculés suivant la formule $\frac{\mathrm{Ni}-(\mathrm{Nu}+\mathrm{Nf})}{\mathrm{Ni}} \times 100$ permettent de mettre en évidence l'utilisation de l'azote de la ration. Les CUP diffèrent significativement pour les 3 séries d'animaux étudiées. Cette différence peut être expliquée en partie par l'azote ingéré ; CUP et quantité d'azote ingérée varient dans le même sens : c'est avec le régime à 8 p. 100 de protéines seul dont le CUP est le plus bas, que l'azote ingéré est le plus faible ( $2 \mathrm{~g} /$ jour). Le CUP de cette série est d'ailleurs négatif comme le laissait supposer la chute de poids relevée pour ces animaux ce qui montre que l'aliment contenant 8 p. 100 de protéines est insuffisant et que l'entretien des lapins nécessite un régime plus riche en azote.

Dans le cas de la série supplémentée par la perfusion d'urée, la différence avec les animaux consommant 16 p. 100 de protéines est due, en outre, au fait qu'une fraction importante de l'azote uréique fourni (près de la moitié) est perdue dans les urines comme le laisse apparaître l'examen des excrétions azotées urinaires. Cependant, la différence notée avec la série nourrie avec 8 p. 100 de protéines et ne recevant pas de perfusion, montre qu'une partie de l'urée perfusée est bien utilisée et constitue un supplément efficace en matière azotée.

2) Par contre, les coefficients d'efficacité protéique $(C E P=\Delta P / N i)$ ne présentent pas de différences significatives entre les séries : bien qu'il soit partiellement utilisé, l'azote de l'urée est aussi efficace que celui des protéines alimentaires.

3) Enfin, si les CEP sont calculés en faisant intervenir l'azote retenu et non plus l'azote ingéré $(\triangle P / N$ retenu), ils se révèlent supérieurs pour les animaux recevant la perfusion d'urée.

L'azote retenu avec le régime supplémenté par l'urée est donc plus efficace que celui de l'aliment contenant 16 p. 100 de protéines; or l'azote uréique est utilisé par la flore cæcale et le lapin, en fait, se nourrit en partie avec les protéines bactériennes ainsi synthétisées. Ce sont elles qui sont responsables de l'élévation du CEP ainsi modifié. Il semblerait donc que les protéines bactériennes soient la forme azotée la plus efficace pour le lapin. 
En conclusion, l'urée a un coefficient d'utilisation protéique inférieur à celui de l'aliment contenant 16 p. 100 de protéines. Ce coefficient d'utilisation varie avec les individus, leur flore cæcale, leur régime alimentaire, les doses d'urée employées et les sources énergétiques fournies, mais il peut être amélioré avec les conditions expérimentales, soit en ralentissant l'uréolyse, soit en augmentant la protéosynthèse bactérienne (Chalupa, 1968).

Par contre, la fraction d'azote uréique utilisée par les bactéries pour synthétiser leur propres protéines qui sont ensuite digérées par le lapin, est très efficace. Les protéines bactériennes apparaissent ainsi comme une source protéique meilleure que l'aliment industriel conventionnel et l'urée peut devenir une source azotée intéressante pour le lapin.

Commission CNERNA Digestion-Absorption, Tours, 13 novembre 1976.

\section{Références}

BARNES R. H., FIALA G., KWONG E., 1963. Decreased growth rate resulting from prevention of coprophagy. Fed. Proc., 22, 125-128.

BATTAGLINI G., 1968. Importanza della coprofagia nel coniglio domestico in rapporto alla utilizzazione di alcuni principi nutrition. Riv. Zoofech. Agric. Vet., 6, 21-37.

BONNAFOUS R., 1973. Quelques aspects de la physiologie colique en relation avec la dualité de l'excrétion fécale chez le lapin. Thèse Doct. d'Etat, Sci. Nat., Université Paul Sabatier, Toulouse.

CHALUPA W., 1968. Problems in feeding urea to ruminants. J. anim. Sci., 27, 207-219.

CHALUPA W., CLARK J., OPLIGER P., LAVKER R., 1970. Detoxication of ammonia in sheep fed soya protein or urea. J. Nutr., 100, 170-176.

FONOLLA J., AGUILERA J., SANZ R., 1972. Influencia del nivel proteico de la duta en el balance de nitrogeno de conejos en crecimiento. Rev. esp. Fisiol., 28, 265-268.

FULLER R., MOORE J. H., 1971. The effect on rabbit intestinal microflora of diets which influence serum cholesterol levels. Lab. animals, 5, 25-30.

HALL E. R., 1952. Investigation on the microbiology of cellulose utilization in domestic rabbits. J. gen. Microbiol., 7, 350-357.

HOOVER W. H., HEITMANN R. N., 1975. Caecal nitrogen metabolism and amino acid absorption in the rabbit. J. Nutr., 105, 245-252.

HSIA Y. E., 1974. Inherited hyperammonemic syndromes. Gastroenterology, 67, 347-374.

JOURNET M., 1973. Les sources d'azote non protéique et leur utilisation. Journées d'information sur l'alimentation azotée des animaux. I. N. R. A. ed.

KOJ A., REGOECZI E., IRONS L., MCFARLANA A. S., 1964. Endogenous catabolism of urea in relation to measurement of plasma protein synthesis rates. Biochem. J., G. B., 91,26 P-27 P.

MARTY J., 1971. Origine et intérêt nutritionnel des acides organiques et des acides aminés dans le cæcum du lapin. Thèse Doct. d'Etat, Sci. Nat., Université Paul Sabatier, Toulouse.

NIEDZWIADEK S., 1976. An attempt at supplementing rabbit feed with urea. 1er Congr. intern. Cunicole, Dijon. Commun. $n^{\circ} 27$.

PROTO V., 1965. Esperienze di coprofagia nel coniglio. Prod. anim., Ital. 4, 1-21.

PROTO V., GIANANI L., 1969. La composizione aminoacidica di alcuni alimenti, delle feci e del caecotrofo nel coniglio sottoposto a differenti diete. Prod. animal. Ital., 8, 203-208.

REGOECZI E., IRONS L., KOJ A., MCFARLANA A. S., 1965. Isotopic studies of urea metabolism in rabbits. Biochem. J., 95, 521-532.

SALSE A., BEAUVILLE M., RAYNAUD P. 1976. Supplémentation d'un régime d'entretien par perfusion intracæcale d'urée chez le lapin. 1 er Congr. intern. Cunicole, Dijon, Commun. no 53. 
SLADE L. M., ROBINSON D. W., 1970. Nitrogen metabolism in rabbits and guinea-pigs. J. anim. Sci., 30, 1044.

SMITH H. W., CRABB W. E., 1961. The fecal bacterial flora of animal and man : its development in the young. J. Path. Bact., 82, 53-66.

SMITH H. W., 1965. Observations on the flora of the alimentary tract of animals and factors affecting its composition. J. Path. Bact., 89, 95-122.

YOSHIDA T., PLEASANTS J. R., REDDY B. S., WOSTMANN B. S., 1971. Aminoacid composition of cæcal contents and feces in germ free and conventionnal rabbits. J. Nutr., U. S. A., 101, 14231429. 